\title{
A Novel Insertion in Exon 23 of the TCOF1 Gene in a Newborn Infant with Treacher Collins Syndrome
}

\author{
Ji Hyeon Yang, Hyo Hyun Cha and Hye Sun Yoon* \\ Department of Pediatrics, Eulji General Hospital, College of Medicine, Eulji University, Seoul, Korea
}

\begin{abstract}
Treacher Collins syndrome (TCS) is the most common and well known mandibulofacial dysostosis with characteristic clinical features including downward slanting of palpebral fissures, coloboma of the lower eyelid, hypoplastic zygomatic arches, micrognathia, macrostomia, microtia, and other deformities of the ears. TCS is caused by mutations in at least 3 genes involved in pre-rRNA transcription: TCOF1, POLR1D and POLR1C. We experienced a 1-day-old female infant with characteristic clinical features of TCS. A novel, heterozygotic mutation within the TCOF1 gene (c.3874_3875insG, p.Ala1292Glyfs*30) was identified to cause a premature stop codon.
\end{abstract}

Key words: Treacher Collins syndrome, TCOF1, Mutation

\section{Introduction}

Treacher Collins syndrome (TCS; OMIM \# 154500) is the most common mandibulofacial dysostosis, which occurs in 1:50,000 live births. It exhibits autosomal dominant inheritance with a high degree of penetrance and variable phenotypic expression. Characteristic clinical features include downward slanting of palpebral fissures, coloboma of the lower eyelid with lack of eyelashes medial to the defect, hypoplastic zygomatic arches, micrognathia, macrostomia, microtia, and other deformities of the ears. Conducting hearing loss and cleft palate are often present. TCS results from mutations in the TCOF1 gene on 5q32-q33.1, ${ }^{1)}$ which encodes the serine/alanine-rich protein named Treacle. ${ }^{2)}$ Mutations observed in TCS result in the introduction of a premature termination codon that can lead to truncation of the protein or to nonsense-mediated mRNA decay. ${ }^{3,4)}$ This suggests that the developmental anomalies result from haploinsufficiency of the TCOF1 gene. ${ }^{5)}$ Recently, Dauwerse et al. conducted a genome- wide copy number analysis on the patients who were negative for a TCOF1 mutation. They presents two additional susceptibility genes related to this disorder-POLR1D,POLR1C genes, which encode subunits of RNA polymerases I and III, account for approximately 9\% of non-TCOF1-related TCS. ${ }^{6}$ We examined a newborn infant with suspected TCS. Genetic testing for the TCOF1 gene was performed, and a novel, heterozygotic mutation (c.3874_3875insG, p.Ala1292Glyfs*30) in exon 23 of the TCOF1 gene was identified. In Korea, 9 cases were reported so far, however genetic testing for TCS was not performed in any cases. This is the first TCS case who diagnosed molecularly in Korea. Here, we report the above case along with a brief review of the literature.

\section{Case Report}

We present the case of a newborn female infant with facial and auricular malformation, who was referred to our center. Pregnancy and family history were uneventful, and vaginal birth

\footnotetext{
Received: 9 August 2013, Revised: 13 September 2013, Accepted: 23 September 2013, Published: 31 December 2013

*Corresponding author: Hye Sun Yoon, M.D.

Department of Pediatrics, Eulji General Hospital, College of Medicine, Eulji University, 280-1, Hagye-1 Dong, Nowon-Gu, Seoul 139-872, Korea

Tel: +82-2-970-8225, Fax: +82-2-970-8862, E-mail: yhs3211@eulji.ac.kr

Conflict of interest: We declare that we do not have any conflicts of interest.

(c) This is an open-access article distributed under the terms of the Creative Commons Attribution Non-Commercial License (http://creativecommons.org/licenses/by-nc/3.0/) which permits unrestricted non-commercial use, distribution, and reproduction in any medium, provided the original work is properly cited.

(c) Copyright 2013 by the Korean Society of Medical Genetics 
occurred at $38^{+3}$ weeks of gestation. She was the first child and at birth, weighed 3,080 $\mathrm{g}$ (50th percentile), with a length of 48.5 $\mathrm{cm}$ (50th percentile) and a head circumference of $33 \mathrm{~cm}$ (25-50th percentile). Apgar scores were 7 at $1 \mathrm{~min}$ and 9 at $5 \mathrm{~min}$. Vital signs at admission were stable, and, especially, there was no signs of respiratory difficulty. Clinical features included downward slanting of the palpebral fissures, blepharophimosis, hypoplasia of the zygomatic bones, mild macrostomia, micrognathia and retrognathia, microtia and hypoplasia of the middle ear on the right side. The chest showed symmetric expansion and lung sound was clear, the abdomen is soft and flat and abnormal structure of extremities was not found. Auditory brainstem response (ABR) and otoacoustic emissions (OAE) tests were performed to evaluate hearing ability and bilateral conductive hearing loss was observed. To evaluate any concomitant anomalies, echocardiography, abdomen sonography, and an ophthamalogic examination were performed, and the results showed no abnormal lesions. Genetic testing was performed to diagnose TCS molecularly. DNA was isolated from peripheral blood leukocytes of the patient. The coding regions and intron/exon boundaries of the TCOF1 gene were amplified by polymerase chain reaction (PCR) by using specific primers under optimal conditions. The analysis demonstrated a novel heterozygotic mutation, c.3874_3875insG, p.Ala1292Glyfs*30, in exon 23 of the TCOF1 gene (Fig. 1), which was found to be a frameshift mutation resulting in a premature stop codon 30 amino acids after the mutation. TCS was molecularly confirmed. Appropriate institutional review board approval for this study was obtained from the Ethics Committee at Eulji General Hospital. Written informed consent was obtained before blood draws for the collection of sample and subsequent analysis. However, we didn't perform the genetic testing and counseling for parents due to refusal. The patient was discharged at 7 days after birth wihout respiratory and feeding problem.

\section{Discussion}

TCS is an autosomal dominant disorder that affects craniofacial morphogenesis during early embryogenesis. ${ }^{7)}$ It is thought to be caused by impaired development of structures derived from the first and second brachial arches. TCS is also known as Franceschetti-Klein syndrome and mandibulofacial dysostosis.

In approximately $81-93 \%$ of patients, TCS is caused by mutations in the TCOF1 gene. ${ }^{4,8)}$ Interfamilial and intrafamilial phenotypic variability is high and there are no direct genotypephenotype correlations in cases of TCOF1 mutations. ${ }^{8}$ The syndrome results from sporadic mutations in $60 \%$ of cases, whereas it has a familial history in the other $40 \% .^{9}$ Thus far, more than 240 mutations in the TCOF1 gene causative of TCS have been described along 28 exons. ${ }^{5,10)}$ The TCOF1 gene mutations include small deletions (49\%), missense/nonsense (22\%), small insertions (12\%), splicing (10\%), gross deletion (3\%), small indels $(2 \%){ }^{10)}$ Nucleotide changes were divided in three functional categories: those that produce a premature stop codon, missense changes, and changes at splice sites. Pathogenic mutations in the TCOF1 gene were reported throughout its coding region and, with the exception of a 5-bp deletion in exon 24 , are usually family-specific. ${ }^{11)}$ The majority of mutations responsible for TCS are localized in exons, mainly in hot spots in exons 10, 15, 16,23 , and 24 . In a recent study about novel mutations of the TCOF1 gene in European patients with TCS, 12 novel mutations were described ; small deletions (10/12), dupulication (1/12), substitution (1/12). And they confirmed that exon 24 as hot

Normal

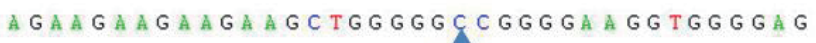

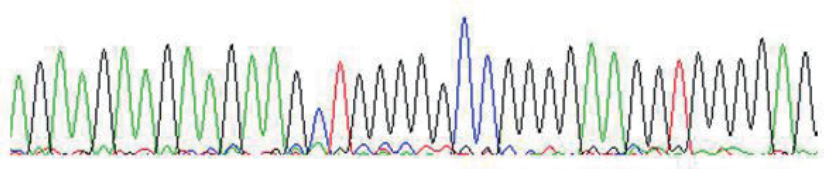

Patient
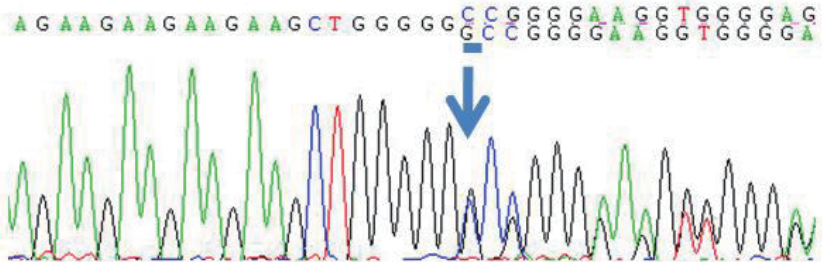

Fig. 1. Partial sequencing of the TCOF1 gene. The patient carries a novel heterozygous mutation, c.3874_3875insG, p.Ala1292Glyfs*30. 
spot of the TCOF1 gene because the c.4366_4370delGAAAA is the most frequent mutation they found it in 3 of 16 affected patients. That is probably due to the high repetition of adenines making exon 24 region prone to polymerase slippage in DNA replication. ${ }^{12)}$ The TCOF1 gene encodes the nucleolar phosphoprotein, Treacle, which is involved in ribosomal biogenesis and shows peak expression in the neural crest cells of the brachial arches. ${ }^{13)}$ Haploinsufficiency of the TCOF1 gene leads to reduction in Treacle levels and thus insufficient ribosome biogenesis, thereby restricting the cell cycle progression of these highly proliferative cell populations, which causes reduced proliferation, cell cycle arrest, and high rates of apoptosis. ${ }^{14)}$ General cranioskeletal hypoplasia occurs due to generation of insufficient neural crest cells.

In Korea, Kim et al. ${ }^{15)}$ reported the first TCS case in 1974 and Cho et al. ${ }^{16)}$ reported a case of familial TCS in 24-year-old mother and 2-month-old male baby diagnosed by typical facial appearance. So far, total 9 of case reports published about their characteristic clinical features, respiratory problems such as an anatomical airway abnormality and difficulty of intubation, feeding problem and plastic repair. However genetic testing was not performed in any cases. Thereby, it is difficult to analyze correlation between the phenotype and the genotype in Korea. According to literature, there is lack of correlation between the phenotype and the type, the size and the location of the mutation. ${ }^{8,7)}$

Molecular diagnostics plays a significant role for patients with TCS, in both the prenatal and postnatal stages. However, it is impossible to predict how severely affected a fetus may be because there are no genotype/phenotype correlations. Consequently, ultrasonography is a useful tool for prenatal diagnosis because this technique may provide information regarding the severity of the fetal condition, as well as being useful for evaluating fetal progression. ${ }^{18)}$

As TCS is such a highly complex disease, treatment of individuals with TCS should be tailored to the specific needs of the individual, preferably by a multidisciplinary team consisting of pediatricians, geneticists and craniofacial surgeons. Airway assessment should be the priority, followed by oropharyngeal repair and midface reconstruction. Auricular repair and bone-assisted hearing aid placement should be carried out in late childhood. Finally, definitive skeletal repair should be completed. ${ }^{19)}$

In conclusion, molecular genetic approaches are very useful for confirming TCS in newborn infants clinically suspected with TCS. In cases with familial history of TCS, molecular genetic approaches could be used for prenatal diagnosis.

\section{References}

1. Positional cloning of a gene involved in the pathogenesis of Treacher Collins syndrome. The Treacher Collins Syndrome Collaborative Group. Nat Genet 1996;12:130-6.

2. Trainor PA, Dixon J, Dixon MJ. Treacher Collins syndrome: etiology, pathogenesis and prevention. Eur J Hum Genet 2009;17:275-83.

3. Dixon MJ, Marres HA, Edwards SJ, Dixon J, Cremers CW. Treacher Collins syndrome: correlation between clinical and genetic linkage studies. Clin Dysmorphol 1994;3:96-103.

4. Splendore A, Silva EO, Alonso LG, Richieri-Costa A, Alonso N, Rosa A et al. High mutation detection rate in TCOF1 among Treacher Collins syndrome patients reveals clustering of mutations and 16 novel pathogenic changes. Hum Mutat 2000;16:315-22.

5. Conte C, D'Apice MR, Rinaldi F, Gambardella S, Sangiuolo F, Novelli G. Novel mutations of TCOF1 gene in European patients with Treacher Collins syndrome. BMC Med Genet 2011;12:125.

6. Dauwerse JG, Dixon J, Seland S, Ruivenkamp CA, van Haeringen A Hoefsloot LH, et al. Mutations in genes encoding subunits of RNA polymerases I and III cause Treacher Collins syndrome. Nat Genet 2011;43:20-

7. Martelli-Junior H, Coletta RD, Miranda RT, Barros LM, Swerts MS, Bonan PR. Orofacial features of Treacher Collins syndrome. Med Oral Patol Oral Cir Bucal 2009:14:E344-8.

8. Teber OA, Gillessen-Kaesbach G, Fischer S, Bohringer S, Albrecht B, Albert $A$, et al. Genotyping in 46 patients with tentative diagnosis of Treacher Collins syndrome revealed unexpected phenotypic variation. Eur J Hum Genet 2004;12:879-90.

9. Jones KL, Smith DW, Harvey MA, Hall BD, Quan L. Older paternal age and fresh gene mutation: data on additional disorders. J Pediatr 1975;86:84-

10. Human Gene Mutation Database[Internet]. Cardiff:Human Gene Mutation Database; 2013 [cited 2013 Seb 7]. Available from: http://www. hgmd.cf.ac.uk/ac/gene.php?gene=TCOF1.

11. Masotti C, Armelin-Correa LM, Splendore A, Lin CJ, Barbosa A, Sogayar $M C$, et al. A functional SNP in the promoter region of TCOF1 is associated with reduced gene expression and YY1 DNA-protein interaction. Gene 2005:359:44-52.

12. Splendore A, Fanganiello RD, Masotti C, Morganti LS, Passos-Bueno MR. TCOF1 mutation database: novel mutation in the alternatively spliced exon $6 \mathrm{~A}$ and update in mutation nomenclature. Hum Mutat 2005:25:429-34.

13. Dixon J, Trainor P, Dixon MJ. Treacher Collins syndrome. Orthod Craniofac Res 2007:10:88-95

14. Dixon J, Jones NC, Sandell LL, Jayasinghe SM, Crane J, Rey JP, et al. Tcof1/ Treacle is required for neural crest cell formation and proliferation deficiencies that cause craniofacial abnormalities. Proc Natl Acad Sci US A 2006;103:13403-8. 
15. Kim JP, Ahn M, Kim WC, Jin DS. A case of Treacher Collins Syndrome. J Korean Pediatr Soc 1974;17:20-2.

16. Cho SH, Chung HS, Choi GJ, Lee HJ, Lee KS. A Case of Familial TreacherCollins Syndrome. J Korean Pediatr Soc 1983;26:1215-9.

17. Trainor PA. Craniofacial birth defects: The role of neural crest cells in the etiology and pathogenesis of Treacher Collins syndrome and the potential for prevention. Am J Med Genet A 2010;152A:2984-94.

18. Edwards SJ, Fowlie A, Cust MP, Liu DT, Young ID, Dixon MJ. Prenatal diagnosis in Treacher Collins syndrome using combined linkage analysis and ultrasound imaging. J Med Genet 1996;33:603-6.

19. Chang CC, Steinbacher DM. Treacher collins syndrome. Semin Plast Surg 2012;26:83-90. 